\title{
Low-dose strontium stimulates osteogenesis but high-dose doses cause apoptosis in human adipose-derived stem cells via regulation of the ERK $1 / 2$ signaling pathway
}

\author{
Abudousaimi Aimaiti ${ }^{1}$, Asihaerjiang Maimaitiyiming ${ }^{1}, \mathrm{Xu}$ Boyong ${ }^{1}$, Kaisaier Aji ${ }^{2}, \mathrm{CaO} \mathrm{Li}^{1^{*}}$ and Lei Cui ${ }^{3^{*}}$
}

\begin{abstract}
Background: Strontium is a widely used anti-osteoporotic agent due to its dual effects on inhibiting bone resorption and stimulating bone formation. Thus, we studied the dose response of strontium on osteo-inductive efficiency in human adipose-derived stem cells (hASCs).

Method: Qualitative alkaline phosphatase (ALP) staining, quantitative ALP activity, Alizarin Red staining, realtime polymerase chain reaction and Western blot were used to investigate the in vitro effects of a range of strontium concentrations on hASC osteogenesis and associated signaling pathways.

Results: In vitro work revealed that strontium (25-500 $\mu \mathrm{M}$ ) promoted osteogenic differentiation of hASCs according to ALP activity, extracellular calcium deposition, and expression of osteogenic genes such as runt-related transcription factor 2, ALP, collagen-1, and osteocalcin. However, osteogenic differentiation of hASCs was significantly inhibited with higher doses of strontium (1000-3000 MM). These latter doses of strontium promoted apoptosis, and phosphorylation of ERK1/2 signaling was increased and accompanied by the downregulation of BCl-2 and increased phosphorylation of BAX. The inhibition of ERK1/2 decreased apoptosis in hASCs.

Conclusion: Lower concentrations of strontium facilitate osteogenic differentiation of hASCs up to a point; higher doses cause apoptosis of hASCs, with activation of the ERK1/2 signaling pathway contributing to this process.
\end{abstract}

Keywords: Strontium, Human adipose-derived mesenchymal stem cells, Osteogenesis, ERK1/2 signaling pathway

\section{Background}

Osteoporosis is a common disease with reduced bone density, and as many as half of all postmenopausal women will have an osteoporosis-related fracture during their lifetime [1]. Anti-osteoporotic drugs, including bisphosphonates and estrogen, and selective receptor-modulating drugs reduce fractures by reducing the number, activity, and lifespan of osteoclasts [2]. Compared to these antiosteoporosis drugs, strontium ranelate (SrRan) has been

\footnotetext{
*Correspondence: caolixjbone@163.com; cuileite@aliyun.com

'Department of Joint Surgery, First Affiliated Hospital of Xinjiang Medical University, 137 Li Yu Shan Road, Urumqi, Xinjiang 830054, People's Republic of China

${ }^{3}$ Department of Plastic Surgery, Institute of Medical Science, Beijing Shijitan Hospital Affiliated to Capital Medical University, 10 Tieyi Road, Beijing 100038, People's Republic of China

Full list of author information is available at the end of the article
}

shown to exert its function via promoting bone formation and simultaneously inhibiting bone resorption [3]; this was shown in several large clinical trials confirming the decreased risk of vertebral fractures in postmenopausal women with osteoporosis. SrRan may improve bone formation due to the stimulation of bone marrow stromal cell (BMSC) osteogenic differentiation. Saidak et al. [4] reported that SrRan counteracts age-related switches in BMSCs from osteoblasts to adipocytes via Wnt5a and NFATc/Maf signaling.

Mesenchymal stem cells (MSCs) are multipotent cells that have the potential for regeneration of damaged tissues in bone disease. Adipose-derived MSCs (ASCs) have several advantages, including immunoprivilege, higher yield at harvest, rapid expansion, and greater genetic stability during long-term culture $[5,6]$. Evidence 
suggests that the administration of ASCs accelerates fracture healing and bone regeneration by direct stimulation of osteogenic differentiation or by secretion of high concentrations of cytokines.

$\mathrm{Ca}, \mathrm{P}, \mathrm{Si}$-containing bioactive ceramic dissolution products, and $\mathrm{SrCaPO}_{4}(52.12 \% \mathrm{Sr})$ enhance the proliferation and osteogenic differentiation of rabbit ASCs [7, 8]. Another study $[9,10]$ indicated that Sr-hardystonite-gahnite (Sr-HT-gahnite), a highly porous and biocompatible $\mathrm{Sr}$ zinc containing bioceramic (7.7 $\mathrm{mg} / \mathrm{L} \mathrm{Sr}$ ion), can induce osteogenesis of rat ASCs in vitro and enhance vascularized bone regeneration in vivo. However, SrRan is implicated in venous thromboembolism (VTE) and the risk of nonfatal myocardial infarction in women with postmenopausal osteoporosis [11]. Moreover, SrRan has been associated with significantly greater risk of death and cardiovascular risk compared with other osteoporotic drugs [12]. Thus, we should study the safety of SrRan with respect to osteogenic differentiation of ASCs.

Mitogen-activated protein kinases (MAPKs) regulate various physiological processes, such as cell growth, differentiation, and apoptosis [13]. Extracellular signal-related kinase (ERK) $1 / 2$ is a member of the MAPK family and is involved in the regulation of the cellular response to apoptotic-promoting signals [14]. Studies suggest that aberrant ERK activation can promote apoptosis under certain conditions, and our work suggests that osteogenic differentiation of human ASCs (hASCs) is mediated by ERK activation [15]. However, how ERK signaling functions during osteogenic differentiation of ASCs stimulated by SrRan is unclear. Thus, we studied the effect of SrRan over a range of doses on osteogenic differentiation of hASCs and assessed SrRan cytotoxicity.

\section{Methods}

\section{Patients and ethics approval}

Fresh human lipoaspirates were obtained from five healthy individuals (average age of 35 years) who received abdominal liposuction at the Department of Plastic and Reconstructive Surgery in Xinjiang Medical University First Affiliated Hospital (Xinjiang, China). Before surgery, all the patients offered written informed consent, and the protocols for human tissue handling were approved by Xinjiang Medical University First Affiliated Hospital.

\section{Cell culture}

Isolation and culture of hASCs was performed according to a previous work [16]. In brief, after washing with an equal volume of $0.1 \mathrm{M}$ phosphate-buffered saline (PBS; $\mathrm{pH} 7.4$ ), lipoaspirates were digested with $0.075 \%$ collagenase type I (Washington Biochemical Corp.) at $37^{\circ} \mathrm{C}$ for $60 \mathrm{~min}$. The digested samples were centrifuged at $1200 \times \mathrm{g}$ for $10 \mathrm{~min}$ to obtain a high-density stromal vascular fraction (SVF), and they were resuspended in low-glucose Dulbecco's modified Eagle's medium (LG-DMEM) containing 10\% fetal bovine serum (FBS), $100 \mathrm{mg} / \mathrm{mL}$ streptomycin, and $100 \mathrm{U} / \mathrm{mL}$ penicillin (growth medium (GM)). They were plated at $4 \times$ $10^{4}$ cells $/ \mathrm{cm}^{2}$ in $100-\mathrm{mm}$ culture dishes (Falcon). When they reached $70-80 \%$ confluence, the cells were passaged and hASCs before the third passage were used in the following study.

Osteogenic differentiation of hASCs was conducted using cultures of GM supplemented with $0.01 \mu \mathrm{M} 1,25$ dihydroxyvitamin D3, $50 \mu \mathrm{M}$ ascorbate-2-phosphate, and $10 \mathrm{mM} \beta$-glycerophosphate. For adipogenic differentiation, the cells were cultured in adipogenic medium (AM) supplemented with $0.5 \mathrm{mM}$ isobutyl-methylxanthine (IBMX), $0.1 \mu \mathrm{M}$ dexamethasone (Dex), $10 \mu \mathrm{M}$ insulin, and $200 \mu \mathrm{M}$ indomethacin in growth medium. The effect of SrRan on the osteogenic differentiation of hASCs was studied using $\mathrm{Sr}(0,25,100,250,500,1,000,1500$, and $2000 \mu \mathrm{M})$ in osteogenic medium $(\mathrm{OM})$.

\section{Alkaline phosphatase staining and activity assay}

At day 10 after induction, alkaline phosphatase (ALP) staining was performed using a leukocyte AP staining kit (System Biosciences). Briefly, the cells were fixed with formalin for $10 \mathrm{~min}$, followed by incubation with BCIP/NBT working solution for $30 \mathrm{~min}$ in the dark at room temperature. After rinsing twice with deionized water, the cells were stained with naphthol AS-MX phosphate for $30 \mathrm{~min}$ in the dark. Excess dye was removed by washing with PBS three times. Positive staining of ALP was observed under a light microscope and quantified with Image-Pro Plus 5.0 software. ALP activity was measured using p-nitrophenylphosphate as the substrate. Absorbance at $405 \mathrm{~nm}$ was measured and the protein in cell lysates was measured using a Bradford assay at $595 \mathrm{~nm}$ on a microplate spectrophotometer (Bio-Rad). ALP activity was normalized according to the total protein.

\section{Accumulated calcium assay}

On day 21 of osteogenic differentiation, the medium was removed and the cells were fixed with $70 \%$ ice-cold ethanol for $1 \mathrm{~h}$, followed by incubation in $40 \mathrm{mM}$ Alizarin Red $\mathrm{S}$ (Sigma) at pH 4.2 for $30 \mathrm{~min}$ at room temperature. After rinsing with fresh PBS and drying at room temperature, the calcium deposits were quantified using a QuantiChrom Calcium Assay Kit (BioAssay Systems). A comparison with an Alizarin Red S dye standard curve was used to evaluate the calcium deposition.

\section{Oil Red $O$ staining}

After 10 days of adipogenic induction, hASCs were stained with Oil Red $\mathrm{O}$ to measure the accumulation of intracellular lipid droplets. Briefly, the cells were fixed 
with $10 \%$ formalin for $30 \mathrm{~min}$, washed in PBS, and stained with a $0.6 \%(\mathrm{w} / \mathrm{v})$ Oil Red O solution for $15 \mathrm{~min}$ at room temperature. After rinsing with deionized water, fat droplet staining was photographed under a phasecontrast microscope (Olympus, Tokyo, Japan).

\section{Live/dead viability staining}

Live and dead cells were measured using an assay kit (Invitrogen) according to the manufacturer's instructions. After incubation with SrRan for 24 and $48 \mathrm{~h}$, hASCs were stained with the live/dead reagent (ethidium homodimer and calcein-AM) at $37^{\circ} \mathrm{C}$ for $30 \mathrm{~min}$. hASC viability was analyzed under a fluorescent microscope (Nikon, Tokyo, Japan).

\section{TUNEL assay}

To measure apoptotic hASCs, terminal deoxynucleotidyl transferase dUTP nick end labeling (TUNEL) staining was used with an In Situ Cell Death Detection Kit (Roche, Mannheim, Germany). Cultured hASCs were fixed with $4 \%$ paraformaldehyde for $1 \mathrm{~h}$ at room temperature,
Table 1 Sequences of primers and real-time polymerase chain reaction conditions

\begin{tabular}{|c|c|c|}
\hline \multicolumn{2}{|l|}{ Gene } & \multirow{2}{*}{$\begin{array}{l}\text { Primer sequences } \\
\text { '-GTCTTACCCCTCCTACCTGA-3' }\end{array}$} \\
\hline$\overline{C b f a 1}$ & Forward & \\
\hline & Reverse & 5'-TGCCTGGCTCTTCTTACTGA-3' \\
\hline \multirow[t]{2}{*}{ ALP } & Forward & 5'-ACGTGGCTAAGAATGTCATC-3' \\
\hline & Reverse & 5'-CTGGTAGGCGATGTCCTTA-3' \\
\hline \multirow[t]{2}{*}{ COL I } & Forward & 5'-TGTTCAGCTTTGTGGACCTC-3' \\
\hline & Reverse & 5'-CTTGGTCTCGTCACAGATCA-3' \\
\hline \multirow[t]{2}{*}{ OCN } & Forward & 5'-CAAAGGTGCAGCCTITGTGTC-3' \\
\hline & Reverse & 5'-TCACAGTCCGGATTGAGCTCA-3' \\
\hline \multirow[t]{2}{*}{$\beta$-actin } & Forward & 5'-ATCATGTTTGAGACCTTCAA-3' \\
\hline & Reverse & 5'-CATCTCTTGCTCGAAGTCCA-3' \\
\hline
\end{tabular}

washed three times with PBS, and then permeabilized in $0.1 \%$ Triton X-100. Fixed cells were exposed to freshly prepared TUNEL reaction mixture for $1 \mathrm{~h}$ in the dark. Cells were then washed with PBS and observed under a fluorescent microscope (Nikon, Tokyo, Japan).
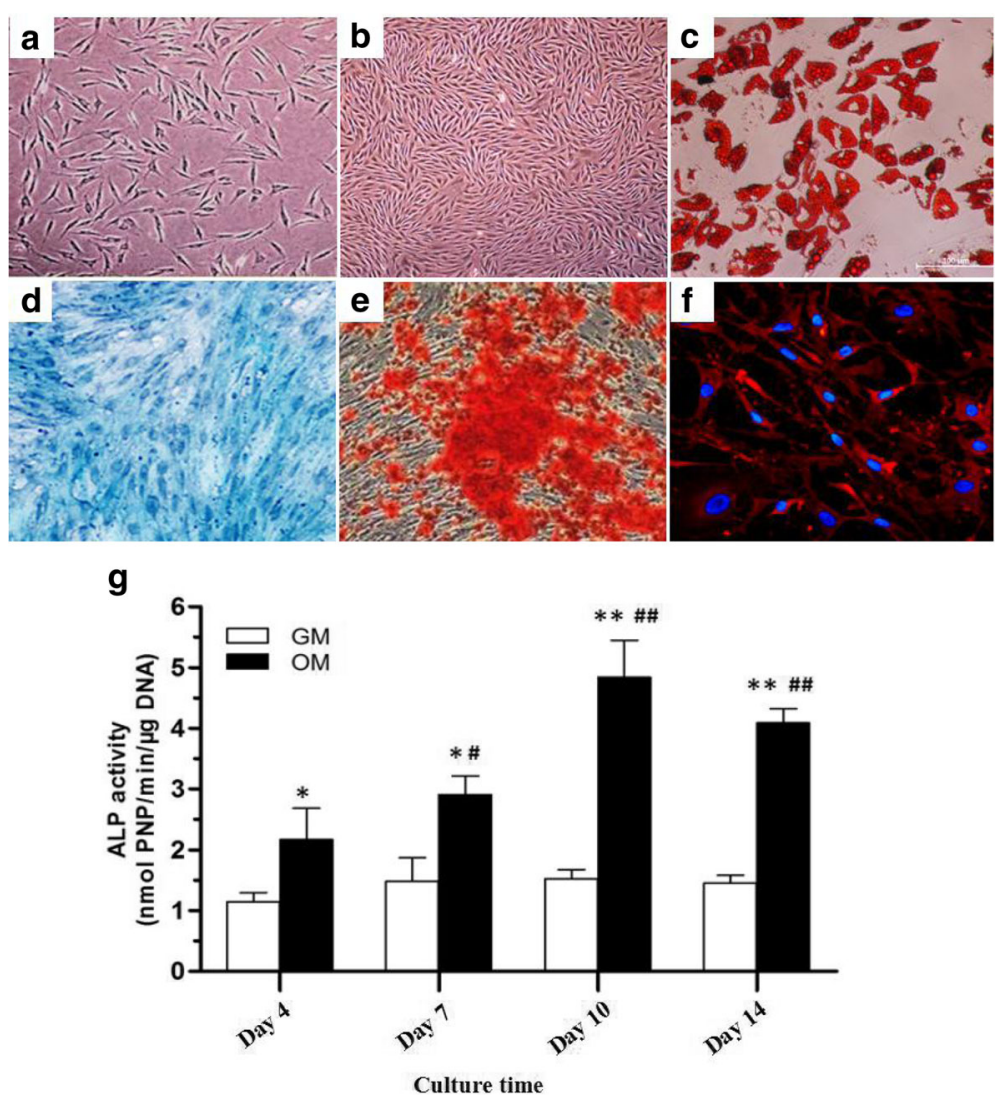

Fig. 1 hASCs had fibroblast-like morphology as observed under a phase-contrast microscope, at passage 0 (a) and 3 (b). Adipogenic differentiation was confirmed with positive Oil Red O staining (c). Osteogenic differentiation was confirmed with positive ALP (d) and Alizarin Red staining (e). Chondrogenic differentiation was verified with immunofluorescent staining for collagen type II (f). ALP activity of hASCs at indicated days after incubation with osteogenic differentiation medium $(\mathbf{g})$ 


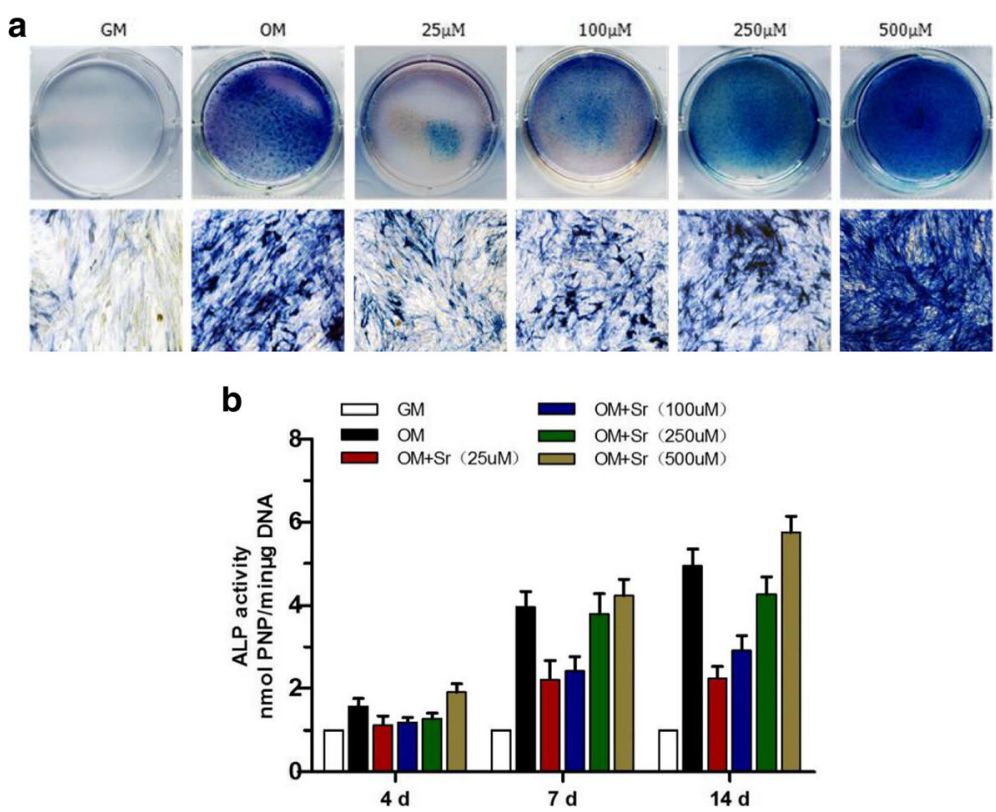

Fig. 2 ALP staining and quantitative assay of osteo-induced hASCs in osteogenic differentiation medium with SrRan. SrRan was added at the indicated concentrations to hASCs in osteogenic differentiation medium induced for 10 days. a ALP expression was measured by staining. b Quantitative ALP activity determined with a colorimetric endpoint assay on the indicated days (d). ALP alkaline phosphatase, GM growth medium, OM osteogenic medium, Sr strontium

\section{Transmission electron microscopy (TEM)}

Cells were cultured in OM medium with/without different doses of SrRan for $48 \mathrm{~h}$. Harvested cells were fixed in ice-cold $2.5 \%$ glutaraldehyde for $2 \mathrm{~h}$. After post-fixing in $1 \% \mathrm{OsO}_{4}$ for $1 \mathrm{~h}$, the samples were dehydrated through an ethanol series, and embedded in epoxy resin. Ultra-thin sections $(60 \mathrm{~nm})$ were double stained with uranyl acetate and lead citrate. Representative areas were examined under TEM (Hitachi, Tokyo, Japan).

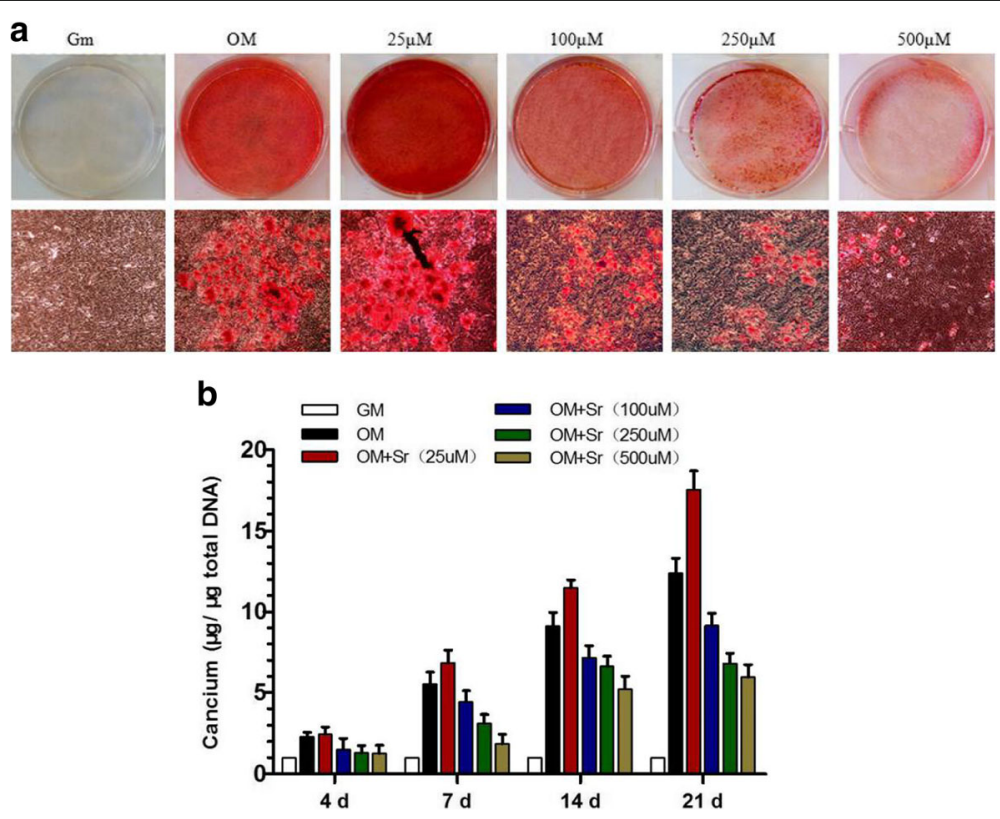

Fig. 3 Effect of SrRan on mineralization of hASCs. a Effect of SrRan added at the indicated concentrations to hASCs in osteogenic differentiation medium. Extracellular calcium deposition was measured with Alizarin Red S staining at day 21. b Extracellular calcium deposition was quantified with a colorimetric method on the indicated days (d). GM growth medium, OM osteogenic medium, Sr strontium 


\section{Quantitative real-time polymerase chain reaction (qRT-PCR)}

Total RNA was isolated using Trizol reagent (Invitrogen) and $2 \mu \mathrm{g}$ of total RNA was reverse-transcribed into $20 \mu \mathrm{l}$ of cDNA. qRT-PCR analysis was performed using the Step One Plus Real-Time PCR System (Applied Biosystems). Primer sequences are shown in Table 1.

\section{Western blot}

For Western blotting, extracted proteins were resolved with sodium dodecyl sulfate polyacrylamide gel electrophoresis (SDS-PAGE) and transferred to a polyvinylidene difluoride (PVDF) membrane (Millipore). After incubation in 5\% bovine serum albumin (BSA) at room temperature, the membranes were incubated with primary antibody at $4{ }^{\circ} \mathrm{C}$ overnight. Horseradish peroxidase (HRP)-conjugated immunoglobulin G (IgG) was used as the secondary antibody. Immunostained protein bands were visualized with an ECL kit (CWBIO, Beijing, China). Protein was quantified and normalized to glyceraldehyde 3-phosphate dehydrogenase (GAPDH).

\section{Statistical analysis}

Data are presented as means \pm standard deviation (SD) from three experiments, and each was an average of the triplicate experiments. SPSS 16.0 software was used for statistical analysis. A paired Student's $t$ test was used to compare groups $(p<0.05$ was considered to be statistically significant).

\section{Results}

\section{Characterization of hASCs}

In the in vitro experiments, hASCs were morphologically homogeneous and fibroblast-like (Fig. 1a and b). Multiple lineage differentiation of hASCs was confirmed by osteogenic, chondrogenic, and adipogenic differentiation (Fig. 1c-f). As shown in Fig. 1g, ALP activity was significantly elevated on days 10 and 14 as indicated by ALP staining and quantitative assays.

\section{SrRan promoted osteogenesis of hASCs}

Figure 2a shows that $\operatorname{SrRan}(25,100$, and $250 \mu \mathrm{M})$ in osteogenic medium decreased ALP staining at 4, 7, and 14 days. At $500 \mu \mathrm{M}$, ALP staining was stronger than in normal osteogenic medium at 14 days. This result is

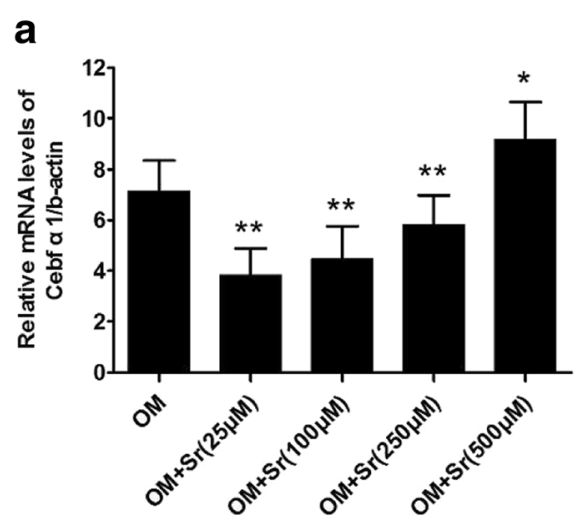

b

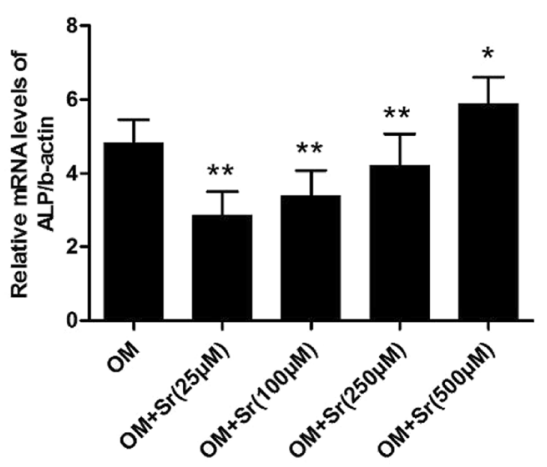

C

d
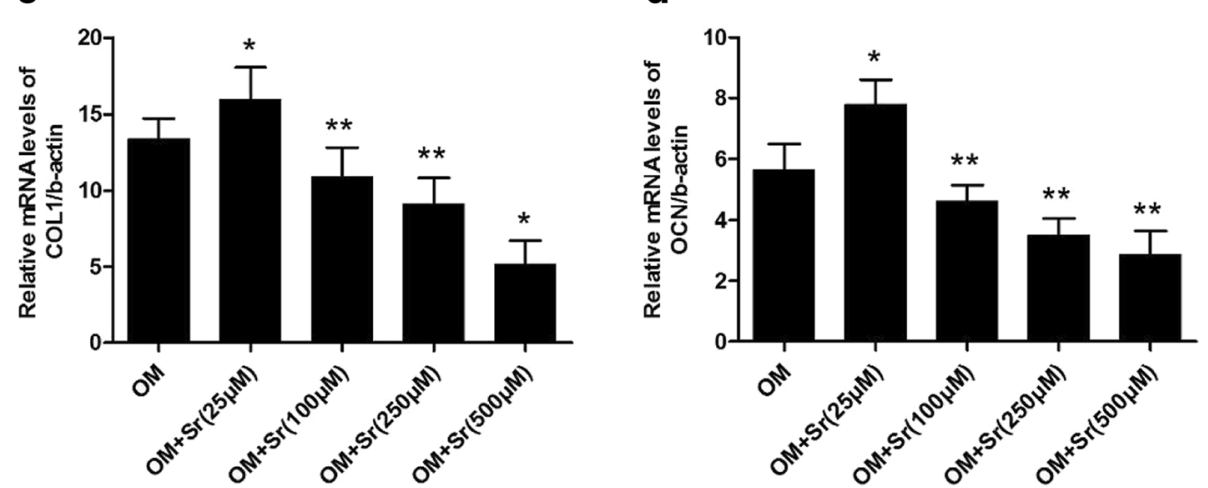

Fig. 4 Effect of SrRan on Cbfa1/RUNX2, ALP, COL I, and OCN gene expression in hASCs. Real-time PCR for osteogenic differentiation-related gene expression of hASCs treated with SrRan $(25,100,250$, and $500 \mu \mathrm{M})$ in OM for 10 days. Gene expression was measured using real-time PCR. a Core binding factor alpha 1 (Cbfa 1); b alkaline phosphatase (ALP); c collagen type I (COLI); and $\mathbf{d}$ osteocalcin (OCN). Cells cultured in OM were used as controls. ${ }^{*} p<0.05,{ }^{* *} p<0.01$ vs. OM. OM osteogenic medium, Sr strontium 


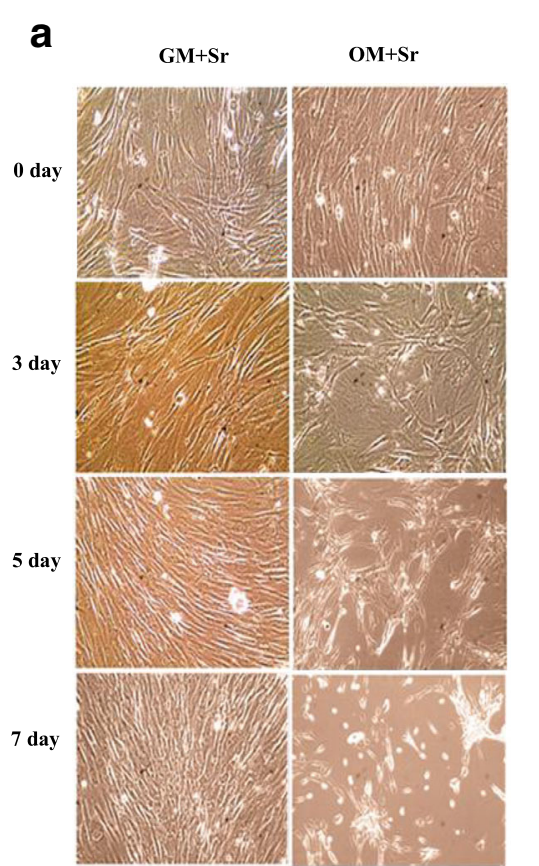

b

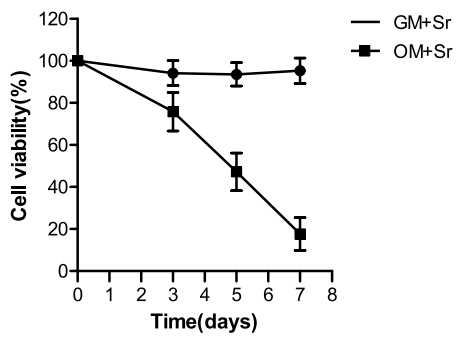

Fig. 5 a Morphological features of hASCs exposed to SrRan at different time points. b hASCs were treated with SrRan (1000 $\mu$ M) in GM and OM at the indicated days and viability was measured with a CCK-8 assay. GM growth medium, OM osteogenic medium, Sr strontium

consistent with the quantitative ALP activity data (Fig. 2b), which shows that ALP activity was suppressed at 25,100 , and $250 \mu \mathrm{M}$ and was enhanced at $500 \mu \mathrm{M}$ after 4, 7, and 14 days. Alizarin Red S staining showed that, at $25 \mu \mathrm{M}$ calcium deposition was significantly increased at 14 and 21 days. At 100, 250, and $500 \mu \mathrm{M}$, calcium deposition was reduced (Fig. 3a and b). qRT-PCR showed that the expression of ALP and runt-related transcription factor 2 (RUNX2) was significantly reduced at 25, 100, and $250 \mu \mathrm{M}$ SrRan, and elevated at $500 \mu \mathrm{M}$

\section{a}
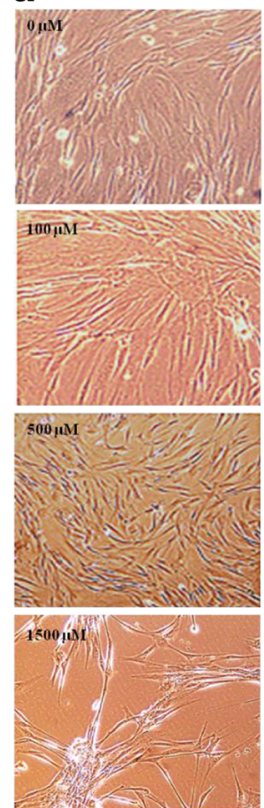

b

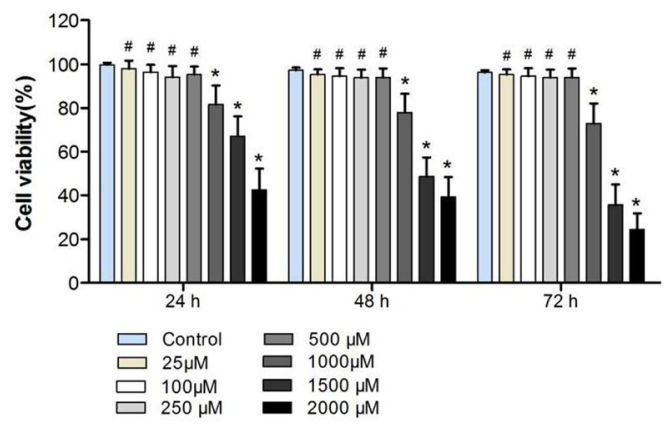

Fig. 6 a Morphological features of hASCs exposed to SrRan. hASCs were treated with SrRan in GM and OM for 3 days. b hASCs exposed to SrRan in GM and OM, with cell viability measured with a CCK-8 assay. $\# p<0.01$ and ${ }^{*} p<0.05$ compared with the control group 
compared with $\mathrm{OM}$ at 10 and 14 days (Fig. 4a and b). In contrast, the expression of collagen-1 (COL-I) and osteocalcin (OCN) was slightly upregulated at $25 \mu \mathrm{M}$ and was significantly suppressed at 100,250 , and $500 \mu \mathrm{M}$.

\section{High-dose SrRan induced apoptosis of hASCs}

We next treated hASCs with $1000 \mu \mathrm{M}$ SrRan for 7 days; cells growing in GM maintained their regular shape and proliferation but SrRan exposure reduced the cell number over 7 days (Fig. 5). Moreover, SrRan at 1000, 1500, and $2000 \mu \mathrm{M}$ inhibited cell proliferation. The cell number was stable with SrRan at 25, 100, 250, and $500 \mu \mathrm{M}$ in OM (Fig. 6). hASCs exposed to SrRan at 1000, 2000, and $3000 \mu \mathrm{M}$ (Fig. 7a) caused shrinkage and more cells to be stained red due to apoptosis at $48 \mathrm{~h}$ (Fig. 7b). TUNEL assay (Fig. 7c and d) showed that
SrRan at 1000, 2000, and $3000 \mu \mathrm{M}$ for $48 \mathrm{~h}$ significantly increased apoptosis.

TEM analysis confirmed apoptotic ultramicrostructural changes, such as cell and nuclear membrane collapse, chromatin loss, and mitochondrial swelling after SrRan $(1000 \mu \mathrm{M})$ for $48 \mathrm{~h}$ (Fig. 8).

\section{The ERK1/2 signaling pathway is involved in high-dose SrRan-induced apoptosis of hASCs}

Western blotting revealed that treatment with SrRan at $1000 \mu \mathrm{M}$ in $\mathrm{OM}$ downregulated $\mathrm{Bcl}-2$ and increased phosphorylation of BAX in hASCs at 8 and $12 \mathrm{~h}$ after induction. Moreover, Fig. 9 shows that $1000 \mu \mathrm{M}$ SrRan treatment induced phosphorylation of ERK as early as 8 and $12 \mathrm{~h}$. The phosphorylation of p38 and JNK was not observed. Consistent with TUNEL and live/dead viability staining data, the apoptosis of hASCs treated with high-

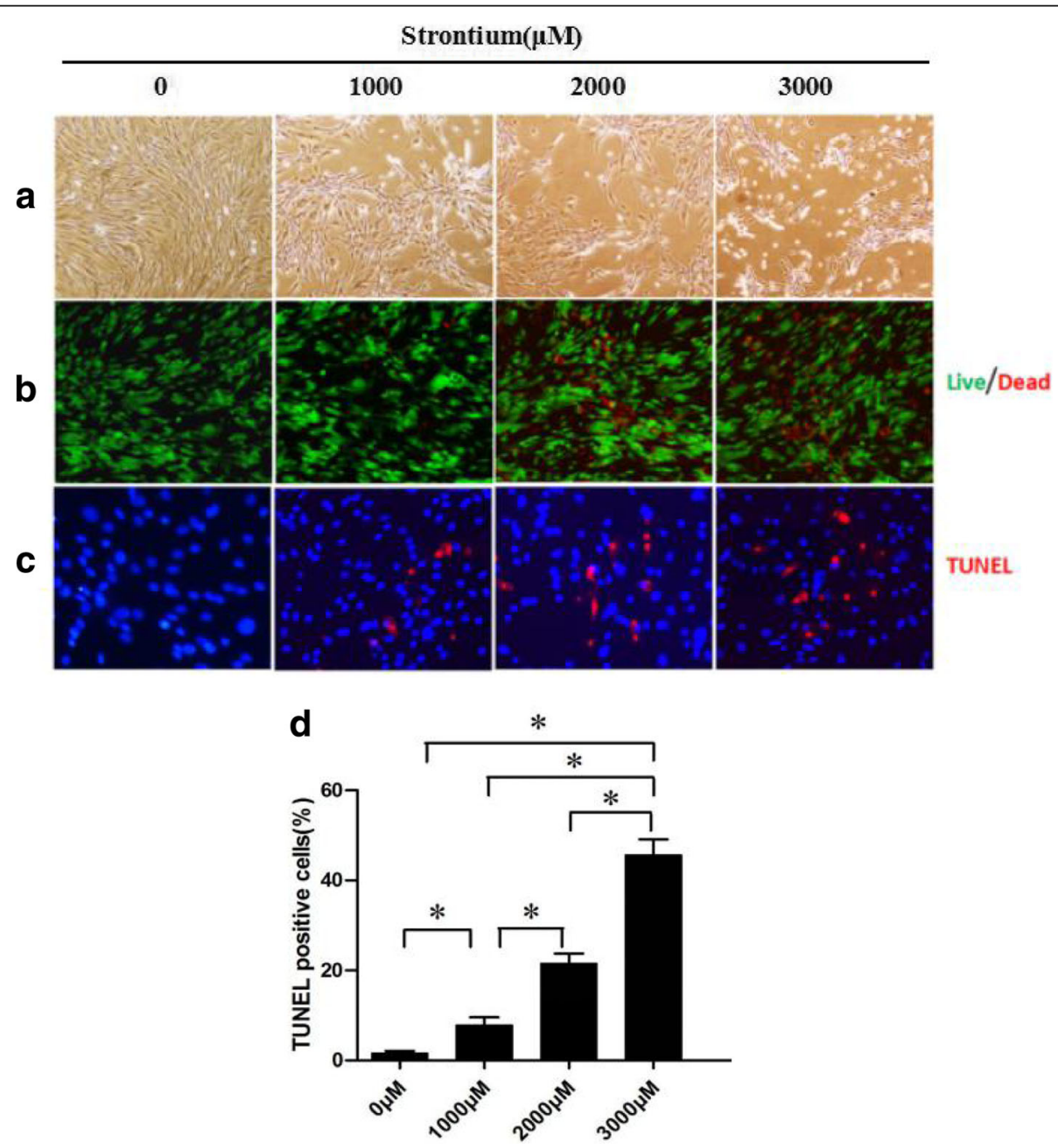

Fig. 7 SrRan-induced apoptotic and morphological changes and decreased hASC viability. a Apoptotic appearance (cell shrinkage) observed in hASCs after exposure to SrRan $(1000,2000$, and $3000 \mu \mathrm{M})$ for $48 \mathrm{~h}$. b High-dose SrRan-treated hASCs were fewer in number compared with the controls according to live/dead staining. c SrRan $(1000,2000$, and $3000 \mu \mathrm{M})$ and apoptosis of hASCs were measured with terminal deoxynucleotidyl transferase dUTP nick end labeling (TUNEL) staining. d Quantification of TUNEL-positive hASCs. * $p<0.05$ compared with the control group 

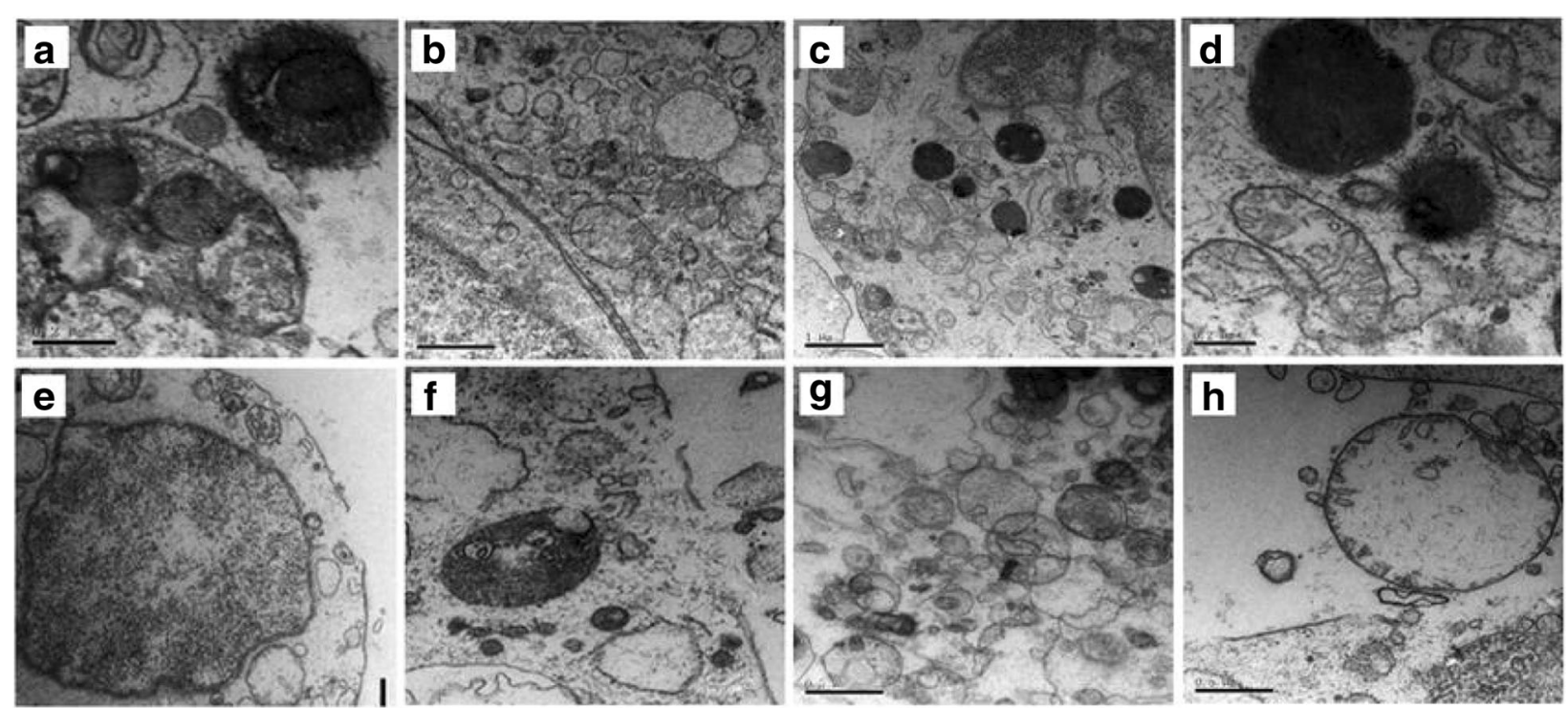

Fig. 8 TEM analysis of hAMSC apoptosis induced by high-dose SrRan (1 mM) for $48 \mathrm{~h}$. a-d Normal control hAMSCs. TEM of the hAMSC group showed intact cell membrane, nuclear membranes, and visible two unit-membranes (a and $\mathbf{b}$ ); cytoplasmic organelle structures were integrated and there was a complete mitochondrial crest (c and $\mathbf{d}$ ). $\mathbf{e}-\mathbf{h}$ Apoptotic hAMSCs from the experimental groups. The cell membrane and nuclear membrane collapsed (e and $\mathbf{f}$ ), and there was cellular content leakage, cytoplasmic organelle elimination, loss of chromatin and mitochondrial swelling, vacuolar degeneration, reduced matrix, and disappearing cristae seen with TEM ( $\mathbf{g}$ and $\mathbf{h})$. Scale bars $=2$ meters

dose SrRan was attenuated by PD98059 (25 $\mu \mathrm{M})$, an inhibitor of ERK pathway activation. The specific JNK inhibitor SP600125 $(10 \mu \mathrm{M})$ and the p38 MAPK inhibitor SB203580 $(5 \mu \mathrm{M})$ had little effect on hASC apoptosis induced by high-dose SrRan (Figs. 10 and 11).

\section{Discussion}

Data show that strontium promotes osteogenesis of mature osteocytes and undifferentiated MSCs in a dosedependent manner at concentrations from 10 to $100,000 \mu \mathrm{M}[17,18]$. However, the effects of low- and high-dose SrRan on osteogenic differentiation of hASCs has not been reported previously. Thus, we investigated the influence of low-dose SrRan on osteogenesis of hASCs at the concentrations used in previous reports [9]. We found that early osteogenic marker expression and ALP activity was increased at $500 \mu \mathrm{M}$ SrRan, but it augmented late osteogenic gene expression and increased calcium deposition at $25 \mu \mathrm{M}$. This result is consistent with Guo et al. [19] who reported that 250$1000 \mu \mathrm{M}$ SrRan caused osteo-induction with the greatest effect being achieved with $250-500 \mu \mathrm{M}$ SrRan. A previous study indicated that significant increases in osteogenic gene expression occurred in MSCs cultured in OM containing $100 \mu \mathrm{M}$ SrRan [20]. Meanwhile, previous studies showed that enhanced osteogenesis occurred in PA20-h5 cell lines treated with SrRan at 50 and $400 \mu \mathrm{M}$ [9]. Canalis et al. [21] reported that $10 \mu \mathrm{M}$ SrRan increased bone formation in rat calvarial cells, and another study showed a significant increase in the osteogenic marker RUNX2 in human MSCs after treatment with SrRan from 2.4-240 $\mu \mathrm{M}$ [22]. Studies have also shown that osteo-induction was achieved at $300 \mu \mathrm{M}$ SrRan in pre-osteoblastic U-33 cells [20]. This is probably because SrRan has a biphasic effect on bone regeneration; dosedependent effects of SrRan at different time points of hASC osteogenesis suggests a complexity of interactions of the effect of cations on bone regeneration. This is in agreement with data from Nardone et al. [9] who showed that ALP production occurred at $400 \mu \mathrm{M}$ SrRan and calcium deposit formation occurred at lower concentrations $(2.5-50 \mu \mathrm{M})$. Another study suggested that SrRan may inhibit calcium deposition in human preadipocytes, indicating that alterations of physicochemical properties in the structure of hydroxyapatite crystals impedes its formation [23]. Accordingly, our results demonstrated that SrRan $(25-500 \mu \mathrm{M})$ promotes hASC osteogenesis in a dose- and time-dependent manner.

However, studies have also shown the opposite results, indicating that SrRan optimally simulates osteogenesis at $1000 \mu \mathrm{M}$ or greater $[24,25]$. The expression of RUNX-2 and ALP increased with SrRan greater than $500 \mu \mathrm{M}$, and therefore we studied the influence of SrRan at high concentrations on the osteogenesis of hASCs and whether high-dose SrRan elicited any adverse effects. We found that the growth of hASCs was significantly suppressed by SrRan at 1000, 1500, and $2000 \mu \mathrm{M}$, and that number of dead hASCs significantly increased at these doses. The TUNEL assay revealed that hASCs exposed to SrRan at these three doses for $48 \mathrm{~h}$ had more apoptotic 

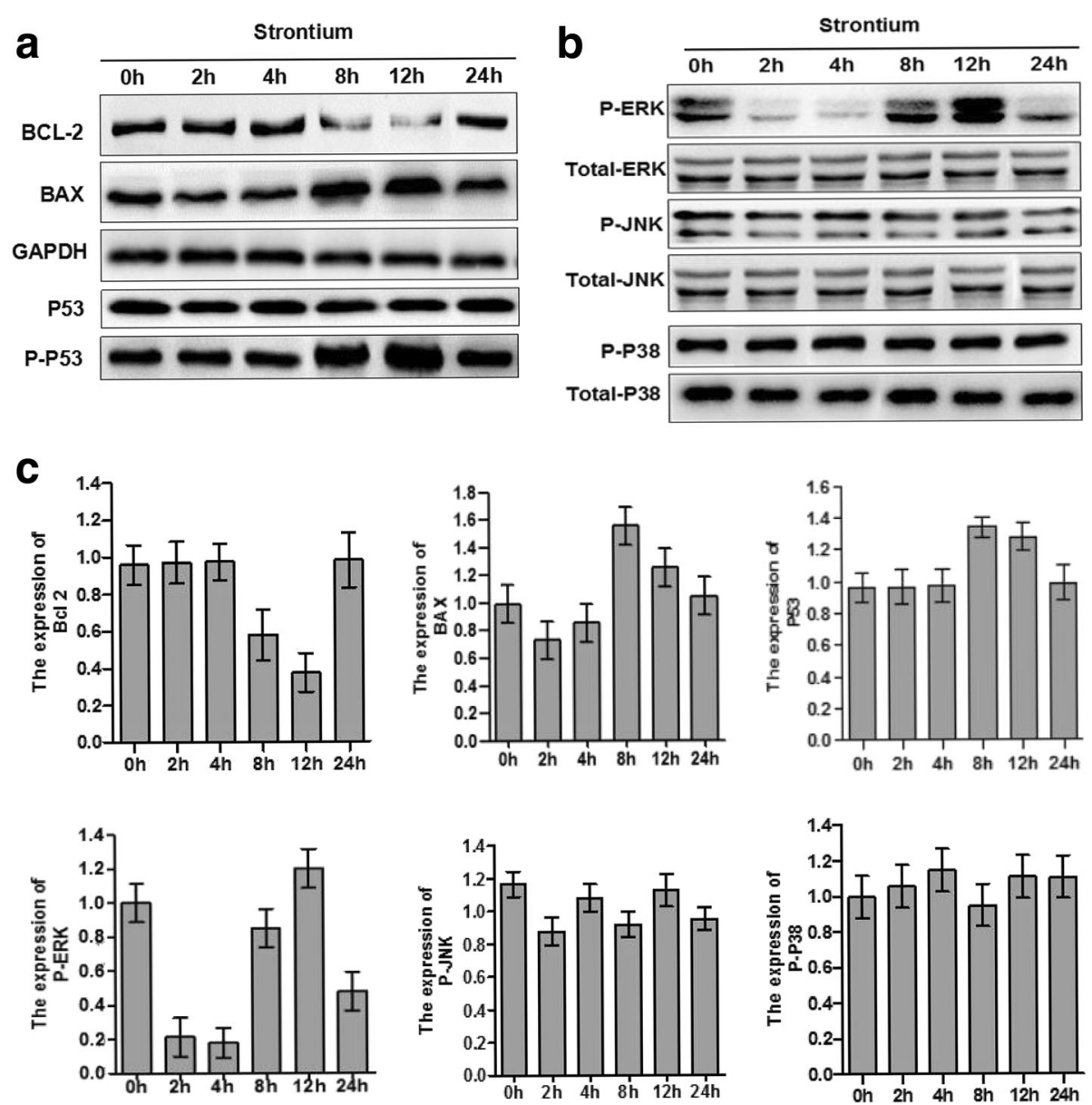

Fig. 9 SrRan activated MAPK pathways mediated by ERK, JNK, p38, and upregulated the expression of Bax and Bcl-2 in hASCs. a SrRan $1000 \mu$ M treatment downregulated BCl-2 protein, and increased the expression of BAX and phosphorylation of P53in hASCs at 8 and 12 h. $\mathbf{b}$ The expression of total and phosphorylated ERK, p38, and JNK was measured by Western blotting in hASCs treated with SrRan $1000 \mu \mathrm{M}$ at the indicated times. Phosphorylation of ERK was significantly increased at 8 and $12 \mathrm{~h}$. No change occurred with phosphorylation of p38 and JNK after 0, 2, 4, 8, 12, and 24 h. c Results of densitometric scans of blots. Data are means \pm SEM for three independent experiments

cells. Furthermore, TEM analysis showed typical apoptotic ultramicrostructural changes in hASCs treated with $1000 \mu \mathrm{M}$ SrRan for $48 \mathrm{~h}$. To our knowledge, this is the first study to show that high-dose $\operatorname{SrRan}(>1 \mathrm{mM})$ causes apoptosis of hASCs under osteogenic differentiation. Of note, some studies show that SrRan at high concentrations promotes osteogenic differentiation of MSCs. Fromigué et al. [26] reported that $3000 \mu \mathrm{M} \mathrm{SrCl}{ }_{2}$ had the greatest osteogenic induction of MC3T3-E1 cells when combined with SrRan. Peng et al. [27] demonstrated that $4000 \mu \mathrm{M}$ SrRan increased the expression of RUNX2 and OCN in human MSCs. These differences may be explained by different cell lineages which respond differently to SrRan at similar concentrations [9, 24, 25]. Zhu et al. [20] showed that $300 \mu \mathrm{M}$ SrRan enhanced osteogenesis of rat MSCs and pre-osteoblastic U-33 cells but it had no obvious effect on OB-6 cells. Furthermore, target genes associated with osteogenesis are variably activated by SrRan in the same cells. SrRan at 0.1-1 mM significantly upregulated BSP and OCN expression, but RUNX2 expression was not changed in osteoblast-like OB6 cells. However, with the stimulation of SrRan at similar concentrations, the expression of RUNX2 was increased in bone marrow MSCs and OCN expression was not affected.

ERK1/2, a member of the MAPK family, is a confirmed pro-survival factor that participates in the regulation of various cell responses, including proliferation, migration, differentiation, and death [28]. ERK activation is noted in apoptosis caused by diverse stimuli $[29,30]$. In our previous work, we reported ERK signaling pathway-mediated osteogenic differentiation of hASCs and adipogenic transition initiated by dexamethasone [16]. Here, we report that with the increased apoptosis of hASCs due to higher doses ( $\geq 1 \mathrm{mM})$ of SrRan, ERK was activated and the inhibition of ERK activation using PD98059 reversed hASC apoptosis. Phosphorylation of 


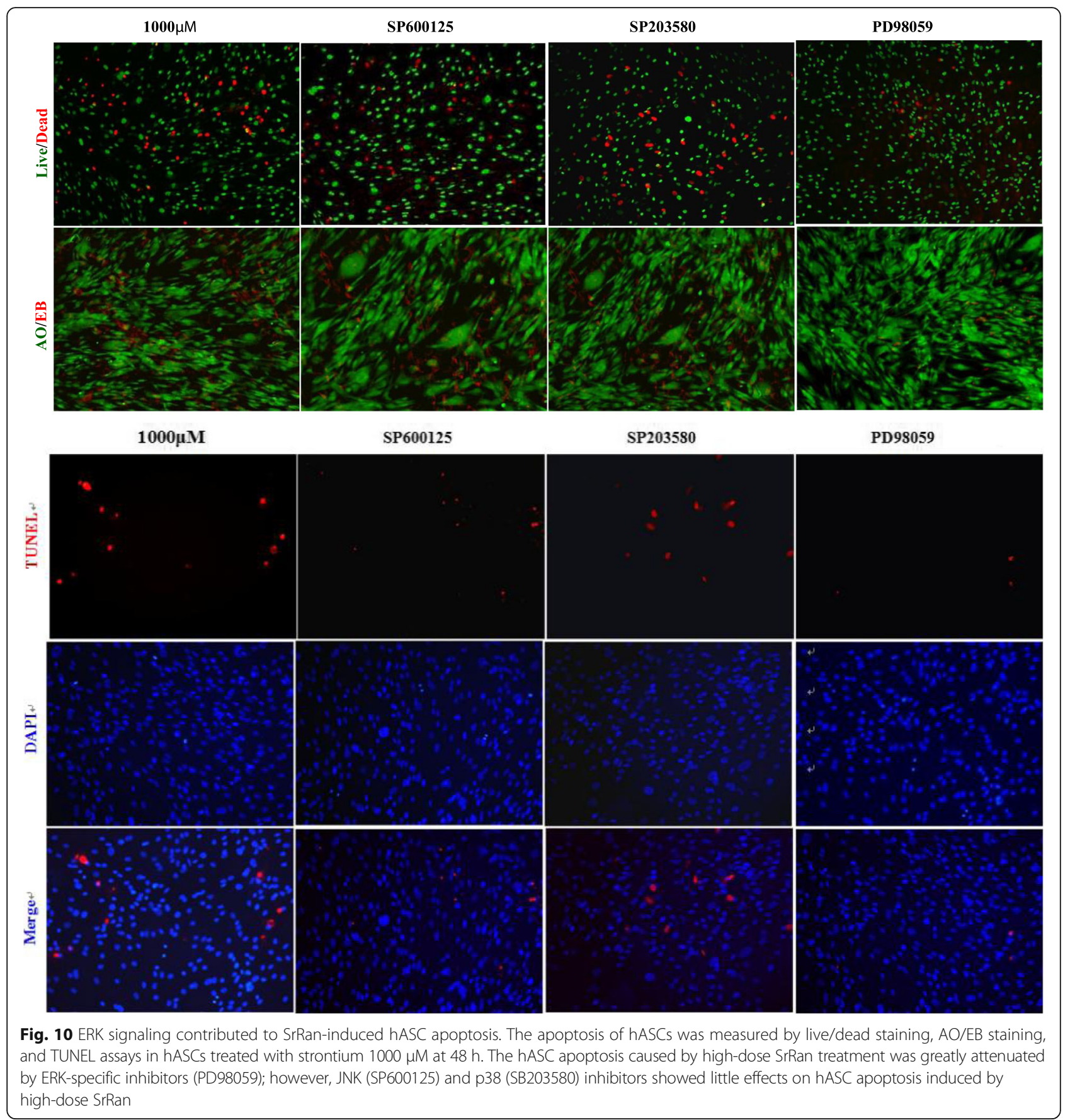

p38 and JNK did not occur and an inhibitor of p38 and JNK had no effect on hASC apoptosis. Thus, the activation of ERK signaling is responsible for high-dose SrRaninduced apoptosis of hASCs and this was not changed by the p38 and JNK pathways. Bcl-2 is a principal family of proteins that protects cells from apoptosis, and Bax induces apoptosis [31]. Thus, the balance between the proand anti-apoptotic Bcl-2 family of proteins influences apoptosis [32]. We found that higher doses of SrRan downregulated Bcl-2 and that the expression of BAX was increased in hASCs. Apoptosis can be initiated by intrinsic or extrinsic pathways and p53 is central to the intrinsic apoptotic pathway [33]. Thus, the phosphorylation of p53 increased in hASCs exposed to high-dose SrRan for 8 and $12 \mathrm{~h}$, suggesting that SrRan-induced apoptosis of hASCs may be modulated by p 53 signaling.

\section{Conclusions}

We suggest that low-dose SrRan enhances hASC osteogenic differentiation and higher doses causes hASC 


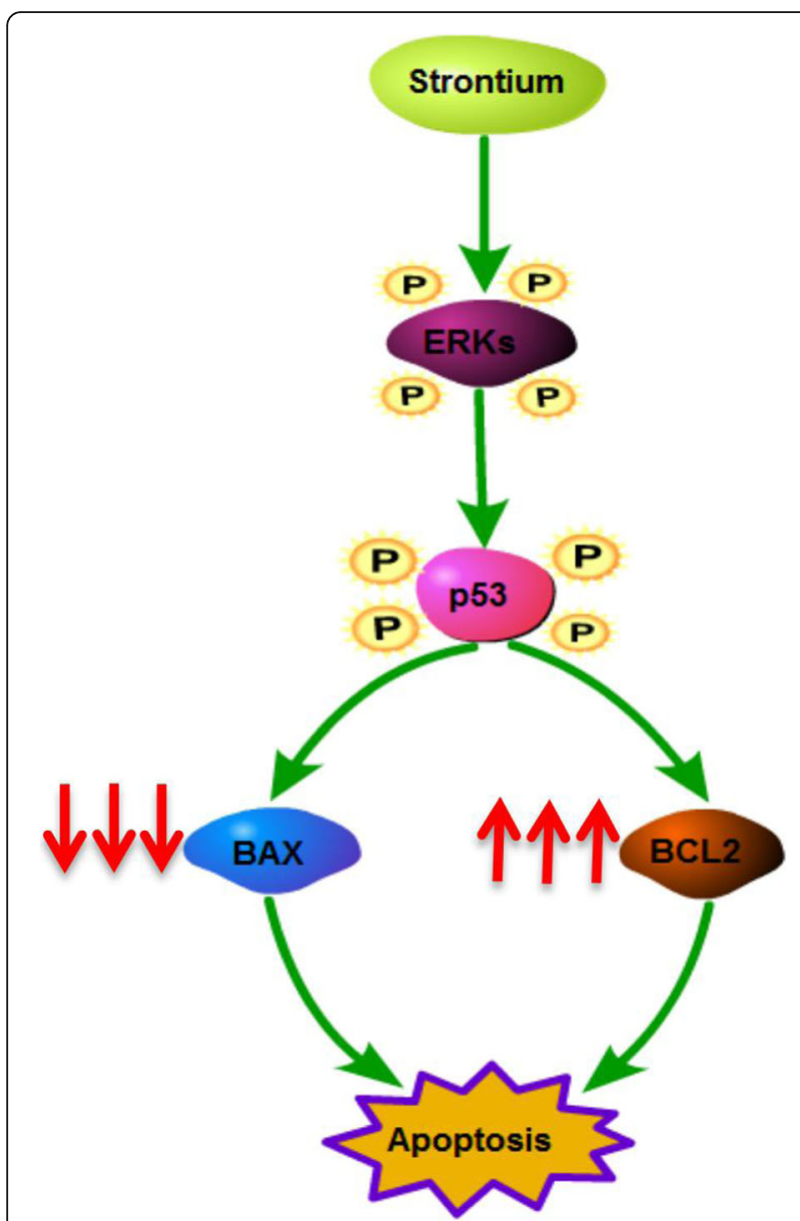

Fig. 11 Diagram of high-dose SrRan-induced hASC apoptosis by ERK/p53-mediated pathways and subsequent Bcl-2 family-mediated mitochondrial apoptosis. SrRan activates ERK phosphorylation, which may increase phosphorylation of its target gene P53. Activation of P53, in turn, downregulates predicted target genes, anti-apoptotic protein, and $\mathrm{BCl}-2$, and upregulates the expression of the pro-apoptotic protein BAX in hASCs in OM, and causes mitochondrial apoptosis

apoptosis via activation of the ERK signaling pathway and subsequent Bcl-2 family-mediated apoptosis. This information may lay the foundation for developing SrRancontaining scaffolds for use in bone tissue engineering.

\section{Abbreviations}

ALP: Alkaline phosphatase; AM: Adipogenic medium; ASC: Adipose-derived mesenchymal stem cell; BMSC: Bone marrow stromal cell; BSA: Bovine serum albumin; COL-1: Collagen-1; Dex: Dexamethasone; ERK: Extracellular signalregulated kinase; FBS: Fetal bovine serum; GAPDH: Glyceraldehyde 3phosphate dehydrogenase; GM: Growth medium; hASC: Human adiposederived mesenchymal stem cell; IBMX: Isobutyl-methylxanthine; LGDMEM: Low-glucose Dulbecco's modified Eagle's medium; MAPK: Mitogenactivated protein kinase; MSC: Mesenchymal stem cell; OCN: Osteocalcin; OM: Osteogenic medium; PBS: Phosphate-buffered saline; PVDF: Polyvinylidene difluoride; qRT-PCR: Quantitative real-time polymerase chain reaction; RUNX2: Runt-related transcription factor 2; SDS-PAGE: Sodium dodecyl sulfate polyacrylamide gel electrophoresis; Sr: Strontium;

SrRan: Strontium ranelate; SVF: Stromal vascular fraction; TEM: Transmission electron microscopy; TUNEL: Terminal deoxynucleotidyl transferase dUTP nick end labeling

\section{Acknowledgements}

None.

\section{Funding}

This work was supported by the National Natural Science Foundation of China (81303065), the National Natural Science Foundation of China-Xinjiang joint fund (U1503221), Major projects in the Xinjiang Uygur Autonomous Region (201430123-3), and the National Natural Science Foundation of China (81471798).

\section{Availability of data and materials}

The data that support the findings of this study are available from the corresponding author upon reasonable request.

\section{Authors' contributions}

AA performed the experiments and wrote the manuscript. AM performed the statistical analysis. XB participated in the design of the study. KA collected and assembled the data. $\mathrm{CL}$ conceptualized and designed the study and provided financial support. LC wrote and approved the final manuscript. All of the authors have approved the final version of the manuscript.

\section{Authors' information}

AA is a joint replacement surgeon and postdoctoral scientist at the First Affiliated Hospital of Xinjiang Medical University. AM and CL are Professors of Joint Surgery and XB is a physician of joint replacement at the First Affiliated Hospital of Xinjiang Medical University. KA is a Ph.D. student at the First Affiliated Hospital of Xinjiang Medical University. LC is a Professor of Plastic Surgery at Beijing Shijitan Hospital affiliated with Capital Medical University.

Ethics approval and consent to participate

All of the patients offered written informed consent before surgery, and protocols for human tissue handling were approved by the Xinjiang Medical University First Affiliated Hospital.

\section{Consent for publication}

The authors consent to publication of all details and images for this manuscript.

\section{Competing interests}

The authors declare that they have no competing interests.

\section{Publisher's Note}

Springer Nature remains neutral with regard to jurisdictional claims in published maps and institutional affiliations.

\section{Author details}

'Department of Joint Surgery, First Affiliated Hospital of Xinjiang Medical University, 137 Li Yu Shan Road, Urumqi, Xinjiang 830054, People's Republic of China. ${ }^{2}$ Department of Urology, The First Affiliated Hospital of Xinjiang Medical University, Urumqi, Xinjiang 830054, China. ${ }^{3}$ Department of Plastic Surgery, Institute of Medical Science, Beijing Shijitan Hospital Affiliated to Capital Medical University, 10 Tieyi Road, Beijing 100038, People's Republic of China.

Received: 7 July 2017 Revised: 10 November 2017

Accepted: 15 November 2017 Published online: 19 December 2017

\section{References}

1. Burge R, Dawson-Hughes B, Solomon DH, Wong JB, King A, Tosteson A. Incidence and economic burden of osteoporosis-related fractures in the United States, 2005-2025. J Bone Miner Res. 2007;22(3):465-75.

2. Milat F, Ebeling PR. Osteoporosis treatment: a missed opportunity. Med J Aust. 2016;205(4):185-90.

3. Meunier PJ, Roux C, Seeman E, Ortolani S, Badurski JE, Spector TD, et al. The effects of strontium ranelate on the risk of vertebral fracture in women with postmenopausal osteoporosis. N Engl J Med. 2004; 350(5):459-68.

4. Saidak Z, Haÿ E, Marty C, Barbara A, Marie PJ. Strontium ranelate rebalances bone marrow adipogenesis and osteoblastogenesis in senescent osteopenic mice through NFATc/Maf and Wnt signaling. Aging Cell. 2012;11(3):467-74. 
5. Cowan CM, Shi YY, Aalami OO, Chou YF, Mari C, Thomas R, et al. Adiposederived adult stromal cells heal critical-size mouse calvarial defects. Nat Biotechnol. 2004;22(5):560-7.

6. Noël D, Caton D, Roche S, Bony C, Lehmann S, Casteilla L, et al. Cell specific differences between human adipose-derived and mesenchymal-stromal cells despite similar differentiation potentials. Exp Cell Res. 2008;314(7):1575-84.

7. Mohan BG, Suresh Babu S, Varma HK, John A, et al. In vitro evaluation of bioactive strontium-based ceramic with rabbit adipose-derived stem cells for bone tissue regeneration. J Mater Sci Mater Med. 2013;24(12):2831-44.

8. Nardone V, Fabbri S, Marini F, Zonefrati R, Galli G, Carossino A, et al. Osteodifferentiation of human preadipocytes induced by strontium released from hydrogels. Int J Biomater. 2012;2012:865291.

9. Nardone V, Zonefrati R, Mavilia C, Romagnoli C, Ciuffi S, Fabbri S, et al. In vitro effects of strontium on proliferation and oteoinduction of human preadipocytes. Stem Cells Int. 2015;2015:871863.

10. Wang G, Roohani-Esfahani SI, Zhang W, Lv K, Yang G, Ding X, et al. Effects of Sr-HT-Gahnite on osteogenesis and angiogenesis by adipose derived stem cells for critical-sized calvarial defect repair. Sci Rep. 2017;7:41135.

11. Abrahamsen B, Grove EL, Vestergaard P. Nationwide registry-based analysis of cardiovascular risk factors and adverse outcomes in patients treated with strontium ranelate. Osteoporos Int. 2014;25(2):757-62.

12. Vestergaard P. New strategies for osteoporosis patients previously managed with strontium ranelate. Ther Adv Musculoskelet Dis. 2014;6(6):217-25.

13. Liu HZ, Yu C, Yang Z, He JL, Chen WJ, Yin J, et al. Tubeimoside I sensitizes cisplatin in cisplatin-resistant human ovarian cancer cells (A2780/DDP) through down-regulation of ERK and up-regulation of p38 signaling pathways. Mol Med Rep. 2011;4(5):985-92.

14. Cargnello M, Roux PP. Activation and function of the MAPKs and their substrates, the MAPK-activated protein kinases. Microbiol Mol Biol Rev. 2011;75(1):50-83.

15. Liu Q, Cen L, Zhou H, Yin S, Liu G, Liu W, et al. The role of the extracellular signal-related kinase signaling pathway in osteogenic differentiation of human adipose-derived stem cells and in adipogenic transition initiated by dexamethasone. Tissue Eng Part A. 2009;15(11):3487-97.

16. Gu H, Guo F, Zhou X, Gong L, Zhang Y, Zhai W, et al. The stimulation of osteogenic differentiation of human adipose-derived stem cells by ionic products from akermanite dissolution via activation of the ERK pathway. Biomaterials. 2011;32(29):7023-33.

17. Brennan TC, Rybchyn MS, Green W, Atwa S, Conigrave AD, Mason RS. Osteoblasts play key roles in the mechanisms of action of strontium ranelate. Br J Pharmacol. 2009:157(7):1291-300

18. Marie PJ. Strontium ranelate: New insights into its dual mode of action. Bone. 2007:40(5):S5-8.

19. Guo X, Wei S, Lu M, Shao Z, Lu J, Xia L, et al. Dose-dependent effects of strontium ranelate on ovariectomy rat bone marrow mesenchymal stem cells and human umbilical vein endothelial cells. Int J Biol Sci. 2016;12(12):1511-22

20. Zhu LL, Zaidi S, Peng Y, Zhou H, Moonga BS, Blesius A, et al. Induction of a program gene expression during osteoblast differentiation with strontium ranelate. Biochem Biophys Res Commun. 2007;355(2):307-11.

21. Canalis $E$, Hott M, Deloffre $P, T$ souderos $Y$, Marie PJ. The divalent strontium salt S12911 enhances bone cell replication and bone formation in vitro. Bone. 1996;18(6):517-23.

22. Sila-Asna M, Bunyaratvej A, Maeda S, Kitaguchi H, Bunyaratavej N. Osteoblast differentiation and bone formation gene expression in strontium-inducing bone marrow mesenchymal stem cell. Kobe J Med Sci. 2007:53(1-2):25-35.

23. Verberckmoes SC, Behets GJ, Oste L, Bervoets AR, Lamberts LV, Drakopoulos $M$, et al. Effects of strontium on the physicochemical characteristics of hydroxyapatite. Calcif Tissue Int. 2004;75(5):405-15.

24. Li Y, Li J, Zhu S, Luo E, Feng G, Chen Q, et al. Effects of strontium on proliferation and differentiation of rat bone marrow mesenchymal stem cells. Biochem Biophys Res Commun. 2012;418(4):725-30.

25. Meunier PJ, Roux C, Ortolani S, Diaz-Curiel M, Compston J, Marquis P, et al. Effects of long-term strontium ranelate treatment on vertebral fracture risk in postmenopausal women with osteoporosis. Osteoporos Int. 2009;20(10):1663-73.

26. Fromigué $\mathrm{O}$, Haÿ E, Barbara A, Marie PJ. Essential role of nuclear factor of activated T cells (NFAT)-mediated Wnt signaling in osteoblast differentiation induced by strontium ranelate. J Biol Chem. 2010;285(33):25251-8.

27. Peng S, Liu XS, Wang T, Li Z, Zhou G, Luk KD, et al. In vivo anabolic effect of strontium on trabecular bone was associated with increased osteoblastogenesis of bone marrow stromal cells. J Orthop Res. 2010;28(9):1208-14.
28. Murphy LO, Blenis J. MAPK signal specificity: the right place at the right time. Trends Biochem Sci. 2006:31:268-75.

29. Nowak G, Clifton GL, Godwin ML, Bakajsova D. Activation of ERK1/2 pathway mediates oxidant-induced decreases in mitochondrial function in renal cells. Am J Physiol Renal Physiol. 2006;291(4):F840-55.

30. Gomez-Sarosi LA, Strasberg-Rieber M, Rieber M. ERK activation increases nitroprusside induced apoptosis in human melanoma cells irrespective of p53 status: role of superoxide dismutases. Cancer Biol Ther. 2009;8(12):1173-82.

31. Markou T, Dowling AA, Kelly T, Lazou A. Regulation of BCl-2 phosphorylation in response to oxidative stress in cardiac myocytes. Free Radic Res. 2009; 43(9):809-16.

32. BBagci EZ, Vodovotz Y, Billiar TR, Ermentrout GB, Bahar I. Bistability in apoptosis: roles of bax, bcl-2, and mitochondrial permeability transition pores. Biophys J. 2006;90(5):1546-59.

33. Sun $X$, Sun GB, Wang $M$, Xiao J, Sun XB. Protective effects of cynaroside against $\mathrm{H} 2 \mathrm{O} 2$-induced apoptosis in $\mathrm{H} 9 \mathrm{c} 2$ cardiomyoblasts. J Cell Biochem. 2011;112(8):2019-29.

\section{Submit your next manuscript to BioMed Central and we will help you at every step:}

- We accept pre-submission inquiries

- Our selector tool helps you to find the most relevant journal

- We provide round the clock customer support

- Convenient online submission

- Thorough peer review

- Inclusion in PubMed and all major indexing services

- Maximum visibility for your research

Submit your manuscript at www.biomedcentral.com/submit

) Biomed Central 